STRUCTURAL SCIENCE CRYSTAL ENGINEERING MATERIALS

ISSN 2052-5206

\section{Intermolecular Interactions in Crystals: Fundamen- tals of Crystal Engineering. Edited by Juan J Novoa. Royal Society of Chemistry, 2017, Pp. 764. Price GBP 99.99 (hardcover). ISBN 978-1-78262-173-7.}

\author{
Gautam R. Desiraju*
}

Solid State and Structural Chemistry Unit, Indian Institute of Science, CV Raman Road, Bengaluru, Karnataka 560012, India. *Correspondence e-mail: gautam.desiraju@gmail.com

Keywords: book review; crystal engineering.

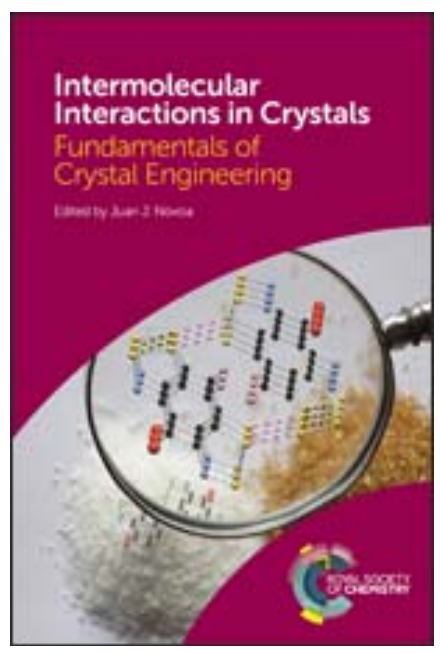

It is very difficult to produce something, which is both 'a textbook for Masters and $\mathrm{PhD}$ students' and 'a reference book for researchers'. So, when I read the preface by the editor, which states these aims, I was quite looking forward to reading the book itself. However, I was disappointed at the end of my enterprise, and doubly so because the book did not appear to me to have succeeded in its aims either as a textbook or as a reference work, barring certain sections.

Any textbook needs to be simple and short, and it should contain non-controversial and easy to understand concepts and principles. The subject matter, including equations and quantitative information, needs to flow in a manner so that the novice learns and at the same time feels impelled to continue reading. A reference work on the other hand contains up to date scholarly material and, in part maybe, even contested and debatable opinions. The features that respectively define a textbook and a reference book are inherently contradictory and in attempting to combine them, the editor was perhaps being unrealistically ambitious.

The title and subtitle of the book are very attractive. There are two issues here: how intermolecular interactions manifest themselves in crystals and how this is connected to the subject of Crystal Engineering. The latter covers both experimental and computational aspects and is now moving into the logical design of properties. The study of intermolecular interactions in crystals, which is indispensable to the subject of Crystal Engineering, is a sub-set of the literature on intermolecular interactions in general. It is this smaller sub-set that ideally should have been covered in this book, also noting that there have been a few textbooks written already. G. A. Jeffrey's An Introduction to Hydrogen Bonding and the multi-author Crystal Engineering. A Textbook by G. R. Desiraju, J. J. Vittal and A. Ramanan come to mind immediately.

In this regard, the initial section of the book, which treats intermolecular interactions per se and not in the context of crystals, was probably not even necessary. Van der Waals interactions and hydrogen bonding have already been treated very well in many other excellent, authoritative texts. The material on these topics in the present book is somewhat repetitive. While the chapter by Novoa attempts to put this matter on track and discusses interactions in the context of crystals, there is confusion in the arrangement and content of other chapters. For example, the chapter by Politzer, Murray and Clark is entitled 'Intermolecular Interactions in Crystals' which is also the title of the book, and this is not a good idea for a multi-author work such as this. To compound the matter further, this is also exactly the title of Section 4 of the book! So, where does the reader go to learn about intermolecular interactions in crystals, the whole book, Politzer et al.'s chapter (which is in Section 3) or to Section 4? This is a difficult choice for a Masters student.

I found chapters 4 (Popelier), 5 (Martin Pendas et al.), 6 (Boto and Contreras-Garcia), 16 (Garcia-Ramos et al.) and 18 (Gatti and Forni) to be a very well written and useful subset of the book itself. I almost got the feeling that a crisp textbook (maybe written by a single author) on charge density methods in Crystal Engineering is the need of the hour because this method is being used increasingly by non-specialists and it addresses the issue of molecules in crystals, even as it calls itself an approach based on atoms in 
molecules. Similarly, the isolated chapter by Bacchi on the use of crystallographic databases is quite useful for both students and researchers because it updates earlier methodologies and at the same time it does not ignore fundamental principles.

As someone who has worked in the area of hydrogen bonding, I felt that the various chapters that deal with this topic in the present book are something of a missed opportunity. There are several standard books on hydrogen bonding even in the context of crystals (Jeffrey and Saenger; Desiraju and Steiner; Gilli and Gilli; Nishio) and the present work could have been an opportunity to bring more focus to this important topic. However, I found many of these hydrogen bonding chapters to be either repeats of what their authors have said in the past or quite preliminary and scattered: beryllium bonds, cation $-\pi$ interactions, hydrogen-hydrogen bonds and multicenter bonds are all undoubtedly important. But do they warrant separate chapters in a book on interactions in crystals and Crystal Engineering? Halogen bonds are another story. These bonds are being actively researched today, and also in the Crystal Engineering context. I would have liked to see a little more coverage of this important topic in this book, including perhaps chalcogen and tetrel bonds.
The final chapter by Braga and Grepioni brings the reader to the practice of Crystal Engineering itself and is well placed in a volume that occurs in an RSC series entitled 'Fundamentals of Crystal Engineering'. Many experimentalists who work in Crystal Engineering are thinking about polymorphs, hydrates and co-crystals. They want to know the properties of important intermolecular interactions that would help them to design crystals with better solubility and permeability properties, if they work, say, with pharmaceuticals. Such researchers and students would constitute the main readership of a book such as this. These readers are not exactly worried about whether Crystal Engineering is 'an area restricted to particular cases' which might 'succeed with educated guessing and a dash of luck', or whether crystal structure prediction is the only real research frontier of tomorrow because it alone deals with the 'extensive, quantitative evaluation of the implied energies'. The study of intermolecular interactions in crystals is now in the chemistry mainstream, and this book succeeds in capturing neither its importance nor its excitement. 